\title{
A stepwise approach to robotic assisted excision of a cesarean scar pregnancy
}

\author{
Payam Katebi Kashi, MD, PhD ${ }^{1}$, Katherine L. Dengler, MD², Eva K. Welch, MD, MS², \\ Angela DiCarlo-Meacham, MD², Araba A. Jackson, MD', G. Scott Rose, MD ${ }^{1}$

\begin{abstract}
'Department of Obstetrics and Gynecology, Inova Fairfax Women's Hospital, Falls Church, VA; '²Department of Obstetrics and Gynecology, Walter Reed National Military Medical Center, Bethesda, MD, USA
\end{abstract}

\section{Objective}

To develop a stepwise approach to robotic assisted excision of cesarean scar pregnancy (CSP) with metroplasty.

Methods

This illustrative video presentation demonstrating CSP, the criteria for ultrasound diagnosis, and a step-by-step approach for robotic assisted excision of CSP and multi-layer hysterotomy closure at a tertiary medical center.

\section{Results}

Robotic assisted resection is a safe and feasible method to treat cesarean scar ectopic pregnancies. Key ultrasonographic characteristics of CSP are highlighted to facilitate its diagnosis, thus allowing for early intervention with a minimally invasive surgical treatment as necessary. Our patient was a 30-year-old gravida 2 para 1 woman with a history of 1 prior-term low transverse cesarean delivery, who presented with vaginal bleeding in the first trimester and was ultimately diagnosed with CSP. After unsuccessful methotrexate therapy, the patient underwent an uncomplicated robotic assisted excision of her CSP and metroplasty in 2 layers using a stepwise approach: Step 1-Creation of a bladder flap; Step 2-Isolation and excision of CSP; Step 3-Hysterotomy closure in 2 layers; and Step 4-Hysteroscopy.

\section{Conclusion}

When diagnosed late, cesarean scar ectopic pregnancy can cause a significant hemorrhage from rupture. Early radiologic diagnosis does not only indicate minimally invasive surgery as a treatment option but also assists with related surgical planning. This video demonstrates a stepwise approach to the robotic assisted excision of CSP with metroplasty. With these 4 simple steps, surgical procedure can be safe and efficient.

Keywords: Robotics; Scar pregnancy; Ectopic pregnancy; Hysterotomy

Cesarean scar pregnancy (CSP) occurs in less than 1\% of ectopic pregnancies and is associated with significant maternal morbidity and mortality due to the risk of hemorrhage [1]. In these pregnancies, the gestational sac or trophoblast is typically located within the dehiscence or niche of the prior cesarean scar. CSP can be detected between days 33 and 94 of pregnancy but is frequently misdiagnosed as normal intrauterine pregnancy, inevitable abortion, gestational trophoblastic disease, or cervical pregnancy [2]. If the pregnancy progresses past the first trimester, an increased risk of a morbidly adherent placenta occurs, including placenta accreta, increta, or percreta [3].

Our video describes a 30-year-old woman, gravida 2 para 1,
Received: 2020.10.15. Revised: 2021.02.08. Accepted: 2021.02.14. Corresponding author: Payam Katebi Kashi, MD, PhD

Department of Obstetrics and Gynecology, Inova Fairfax Women's Hospital, 3300 Gallows Road, Falls Church, VA 22042, USA

E-mail: pkatebi1@gmail.com

https://orcid.org/0000-0002-0138-5868

Articles published in Obstet Gynecol Sci are open-access, distributed under the terms of the Creative Commons Attribution Non-Commercial License (http://creativecommons. org/licenses/by-nc/3.0/) which permits unrestricted non-commercial use, distribution, and reproduction in any medium, provided the original work is properly cited.

Copyright $\odot 2021$ Korean Society of Obstetrics and Gynecology 


\title{
Obstetrics \& Gynecology Science
}

\author{
Vol. 64, No. 3, 2021
}

with a history of a prior low transverse cesarean section, who initially presented to the emergency room due to first trimester vaginal bleeding with a beta human chorionic gonadotropin (bHCG) of $888 \mathrm{mlU} / \mathrm{mL}$. Vaginal ultrasound at that time was non-diagnostic. A repeat bHCG test 48 hours later showed an appropriate increase to $3,100 \mathrm{mIU} / \mathrm{mL}$. A repeat ultrasound at 6 weeks of gestation revealed findings suggestive of a CSP.

High-resolution ultrasound can facilitate CSP diagnosis and allow for an early implementation of an effective and minimally invasive treatment. Seven ultrasound findings have been established to aid in the diagnosis of CSP, including an empty uterine cavity, placenta, or gestational sac embedded in the cesarean scar, a rich vascular pattern around the placenta or sac, a fetal pole with or without a heart rate, a closed internal os and empty endocervical canal, triangular trophoblastic tissue filling the niche scar, and a thin layer of myometrium measuring approximately $2 \mathrm{~mm}$ covering the gestational sac under the bladder [4]. All these ultrasonographic findings were confirmed in our patient.

Several treatment strategies for CSP exist, including methotrexate therapy (systemic or intragestational sac injections), dilation and curettage, hysteroscopic removal, and abdominal excision (robotic, laparoscopic, laparotomy). The success rates for each treatment vary, and the regimen chosen should be based on patient's characteristics and experience of the attending physician. In appropriate patients, conservative management can help avoid the risks and complications associated with surgery. However, in patients at a higher risk of rupture or in patients who do not respond to medical management, an aggressive and early treatment involving surgery should be considered.

Based on ultrasonographic findings of CSP with a mean sac diameter of $12 \mathrm{~mm}$ and no fetal cardiac activity, the patient was extensively counseled and opted for medical management with close surveillance. She received a single dose of methotrexate $\left(50 \mathrm{mg} / \mathrm{m}^{2}\right)$, followed by a two-dose protocol after findings of increased bHCG (from 23,000 to 36,000 $\mathrm{mlU} / \mathrm{mL}$ ) and the presence of fetal cardiac activity. However, a surgery was recommended after failure of 3 doses of methotrexate. Intragestational sac injection of methotrexate or a more aggressive regimen with leucovorin rescue could have been performed; however, these options were not readily available at our institution. A preoperative pelvic magnetic resonance imaging (MRI) performed for surgical planning revealed a $4.1 \mathrm{~cm}$ mass within the cesarean scar niche consistent with a gestational sac.

The patient underwent a robotic excision of her CSP with metroplasty based on a stepwise approach. The first step included the creation of a bladder flap well below the demarcation of the cervix to create adequate space for full excision of the ectopic pregnancy and a multi-layer hysterotomy closure. A uterine manipulator was placed to help delineate the cervix from the lower uterine segment. After the vesicouterine peritoneum was grasped and tented, sharp dissection was performed within the areolar tissue in an avascular dissection plane to facilitate bladder flap creation.

The second step involved the isolation and excision of the CSP. Sharp dissection was performed 360 degrees around the CSP until the bleeding edges of the normal myometrium and cervical tissue were visualized. Given the propensity for hemorrhage occurrence during CSP excision, there are several options to avoid excessive bleeding, including preoperative misoprostol, injection of vasopressin, isolation and temporary uterine artery ligation, and intravenous tranexamic acid [5-7]. Fortunately, these modalities were not necessary in this case. After complete excision, the specimen was removed using a laparoscopic surgery retrieval pouch through an $8 \mathrm{~mm}$ port.

The third step involved the completion of the metroplasty in two layers. The uterine defect was sutured with 2-0 barbed monofilament sutures to prevent occlusion of the internal cervical os in the closure. After the first layer was hemostatic, a second imbricating layer was applied to strengthen the repair with a 0 barbed monofilament suture.

The last step was hysteroscopy performed after the completion of the metroplasty. Given the proximity of the ectopic pregnancy to the internal os, hysteroscopy was performed to ensure cervical patency without obstruction.

Our patient's postoperative course was uneventful, and she was discharged home on postoperative day 1. Pathology revealed an ectopic pregnancy with infarcted chorionic villi. She had a 3-month follow-up MRI, which showed complete resolution of the CSP, and the uterine scar myometrial thickness was $1.1 \mathrm{~cm}$. Postoperative imaging allows for further assessment of the myometrial thickness after a surgical repair. While MRI allows for a thorough evaluation of both the endometrium and myometrium, it can be costly and not widely available. Sonohysterography is a cheaper and more accessible alternative. Sonohysterography has greater sensitivity and specificity in CSP evaluation as the defect size is ex- 


\section{Obstetrics \& Gynecology Science}

Payam Katebi Kashi, et al. Robotic cesarean scar pregnancy excision

aggerated by the increased intrauterine pressure, compared to the ultrasound alone [8].

Our patient conceived 15 months after the surgical procedure. She had an ultrasound-indicated cerclage placed at 14 weeks due to the shortened cervix, with an uncomplicated delivery at 37 weeks via a repeat cesarean section due to cholestasis of pregnancy. There are no documented reports in the literature associating short cervix or cervical insufficiency with CSP excision [9-11]. Indications for cerclage placement remain the same as those in the general obstetric population. In our video, we demonstrated a stepwise approach to robotic assisted excision of CSP using metroplasty. The 4 simple steps we described here can be widely adapted to ensure a safe and efficient surgical treatment of CSP.

\section{Video clip}

Video can be found with this article online at https://doi. org/10.5468/ogs.20311.

\section{Conflict of interest}

The authors have no disclosures or conflicts of interest.

\section{Ethical approval}

This video article was approved by the Institutional Review Board at Fairfax Inova Medical Center, Falls Church, Virginia, under protocol \#16-2244. A written informed consent was obtained from the patient.

\section{Patient consent}

The patient provided a written informed consent for the publication of this manuscript and the related video.

\section{Funding information}

None.

\section{References}

1. Seow KM, Huang LW, Lin YH, Lin MY, Tsai YL, Hwang $J L$. Cesarean scar pregnancy: issues in management. Ultrasound Obstet Gynecol 2004;23:247-53.

2. Pędraszewski P, Wlaźlak E, Panek W, Surkont G. Cesarean scar pregnancy-a new challenge for obstetricians. J Ultrason 2018;18:56-62.

3. Sinha P, Mishra M. Caesarean scar pregnancy: a precursor of placenta percreta/accreta. J Obstet Gynaecol 2012;32:621-3.

4. Timor-Tritsch IE, Monteagudo A, Cali G, El Refaey H, Kaelin Agten A, Arslan AA. Easy sonographic differential diagnosis between intrauterine pregnancy and cesarean delivery scar pregnancy in the early first trimester. Am J Obstet Gynecol 2016;215:225.e1-7.

5. Pirtea L, Balint O, Secosan C, Grigora D, Ilina R. Laparoscopic resection of cesarean scar ectopic pregnancy after unsuccessful systemic methotrexate treatment. J Minim Invasive Gynecol 2019;26:399-400.

6. Schmitt A, Crochet P, Agostini A. Robotic-assisted laparoscopic treatment of residual ectopic pregnancy in a previous cesarean section scar: a case report. J Minim Invasive Gynecol 2017;24:342-3.

7. Persson J, Gunnarson G, Lindahl B. Robotic-assisted laparoscopic surgery of a 12-week scar pregnancy with temporary occlusion of the uterine blood supply. J Robotic Surg 2009;3:53-5.

8. Tulandi T, Cohen A. Emerging manifestations of cesarean scar defect in reproductive women. J Minim Invasive Gynecol 2016;23:893-902.

9. Leone FPG, Ragusa G, Lanzani C, Digrandi M, Ferrazzi E. Cervical cerclage after hysteroscopic metroplasty. Ultrasound Obstet Gynecol 2001;18:88.

10. Huang D, Zhang L, Chen J, Zhang S. The cervical function and pregnancy outcomes after hysteroscopic resection of the complete uterine septum, duplicate cervix and vaginal septum. Laparosc Endosc Rob Surg 2019;2:31-3.

11. Kenda Šuster N, Gergolet M. Does hysteroscopic metroplasty for septate uterus represent a risk factor for adverse outcome during pregnancy and labor? Gynecol Surg 2016;13:37-41. 